\begin{tabular}{|c|l|}
\hline Title & Nonlinear analysis of periodic modulation in resonances of cylindrical and spherical acoustic standing waves \\
\hline Author(s) & Kurihara, Eru; Y ano, Takeru \\
\hline Citation & $\begin{array}{l}\text { Physics of Fluids, 18(11), 117107 } \\
\text { https://doi.org/10.1063/1.2393437 }\end{array}$ \\
\hline Issue Date & 2006-11 \\
\hline Doc URL & http://hdl.handle.net/2115/16865 \\
\hline Rights & Copyright $\odot 2006$ A merican Institute of Physics \\
\hline Type & article \\
\hline File Information & PF18117107.pdf \\
\hline
\end{tabular}

Instructions for use 


\title{
Nonlinear analysis of periodic modulation in resonances of cylindrical and spherical acoustic standing waves
}

\author{
Eru Kurihara and Takeru Yano \\ Division of Mechanical and Space Engineering, Graduate School of Engineering, Hokkaido University, \\ Sapporo 060-8628, Japan
}

(Received 20 June 2006; accepted 11 October 2006; published online 22 November 2006)

\begin{abstract}
The nonlinear resonance of cylindrical acoustic standing waves of an ideal gas contained between two coaxial cylinders is theoretically investigated by the method of multiple scales. The wave motion concerned is excited by a small-amplitude harmonic oscillation of the radius of the outer cylinder, and the formulation of the problem includes the wave phenomenon in a hollow cylinder without the inner one as a limiting case. The spherical standing wave in two concentric spheres is also studied in parallel. The resonance occurs if the driving frequency falls in a narrow band around the linear resonance frequency, and in the weakly nonlinear regime, no shock wave is formed in contrast to the plane wave resonance. A cubic nonlinear equation for complex wave amplitude can then be derived by the method of multiple scales. Using a first integral of the cubic nonlinear equation, we shall demonstrate that the resonant oscillation is accompanied by a periodic modulation of amplitude and phase when the dissipation effect due to viscosity and thermal conductivity is negligible. The period of the modulation varies as the minus two-thirds power of the acoustic Mach number defined at the outer cylinder or sphere and decreases with an increase in the radius ratio of the inner and outer cylinders or spheres. When the dissipation effect is small but not negligible, the modulation is slowly weakened and the resonant oscillation approaches a steady state oscillation, which corresponds to the steady solution examined in earlier works. (C) 2006 American Institute of Physics. [DOI: 10.1063/1.2393437]
\end{abstract}

\section{INTRODUCTION}

Acoustic resonance in fluids has long been studied because a large amplitude sound, which has a wide potential for application, can readily be produced as long as the dissipation effect due to viscosity and thermal conductivity is sufficiently small. The resonance of plane standing waves in a closed tube continues to attract particular attention, ${ }^{1-9}$ leading to the development of the corresponding part of nonlinear wave theory. Of primary concern in the nonlinear plane wave resonance is, from both theoretical and application points of view, the formation of shock waves, which causes the energy dissipation at a shock front and restrains the growth of wave amplitude. In the plane wave resonance with shock waves, therefore, the maximum wave amplitude in the quasisteady state oscillation is limited to $O(\sqrt{M}),{ }^{1-9}$ where $M$ is the acoustic Mach number defined at the sound source and usually rather small compared with unity (typically $M \lesssim 10^{-3}$ ).

The weakly nonlinear resonances of cylindrical and spherical standing waves are, on the other hand, shock free if $M$ is not too large. ${ }^{10-13}$ This is because the excitation of higher harmonics required for shock formation is impeded by the fact that the resonance frequency of each $n$th mode $(n=2,3, \ldots)$ is not equal to the $n$ multiple of that of the fundamental mode in cylindrical and spherical standing waves, as sometimes called dissonant. ${ }^{14}$ As a result, the maximum wave amplitude in the shock free resonances of cylindrical and spherical standing waves attains $O\left(M^{1 / 3}\right),{ }^{10-13}$ which can be considerably large for $M \ll 1$ compared to the case of plane waves. The dissonant effect has been utilized for realization of large amplitude and shock free resonant oscillations. ${ }^{15,16}$

The steady state oscillations of shock free resonances in a spherical shell and in an infinitely long cylinder were studied by Chester ${ }^{10}$ and Ellermeier, ${ }^{11,12}$ respectively. However, since they started the analyses from assuming the steady state, the temporal evolutions of wave motions were not clarified. Recently, we have carried out the numerical analysis of resonance of cylindrical standing waves in an ideal gas between two coaxial cylinders, where the wave motion is excited from an initial quiescent gas by a harmonic oscillation of the radius of outer cylinder. ${ }^{13}$ We have found that the shock free resonance is accompanied by a periodic modulation in amplitude and phase from the numerical solutions of the systems of Euler and Navier-Stokes equations. We have also shown that the period of modulation is approximately proportional to $M^{-2 / 3}$ and it is a decreasing function of the radius ratio of the inner and outer cylinders. ${ }^{13}$ Furthermore, a critical acoustic Mach number, above which a shock wave is formed, has been determined numerically. ${ }^{13}$

In the present paper, we perform a theoretical analysis of the modulation of shock free resonances of cylindrical standing waves in two coaxial cylinders and spherical standing waves in two concentric spheres. The problem setup of two coaxial cylinders and two concentric spheres effectively works to address the geometrical effect on the resonances by changing the radius ratio. Restricting ourselves to the weakly nonlinear and shock free regime, we can apply an asymptotic expansion in a small parameter $\epsilon$ to the problem, where $\epsilon$ is 


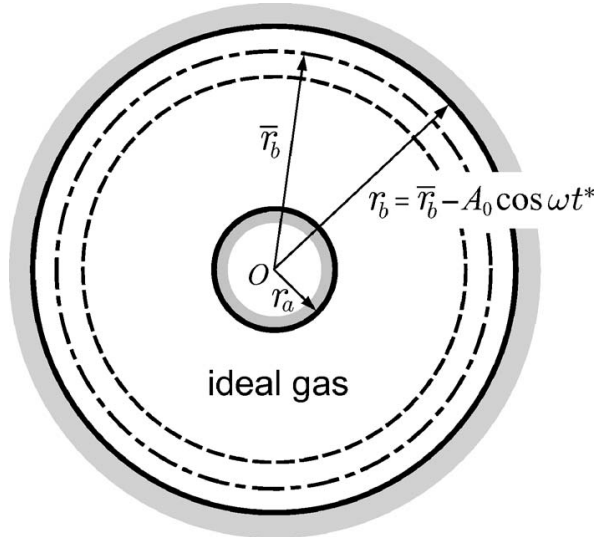

FIG. 1. Schematic of coaxial cylinders or concentric spheres. The acoustic standing wave is excited by a harmonic oscillation of the radius of the outer cylinder or sphere with an amplitude $A_{0}$ and angular frequency $\omega$.

set as the maximum wave amplitude $M^{1 / 3}$. In addition to a characteristic time scale $\omega^{-1}$, where $\omega$ is an angular frequency of oscillation of radius of the outer cylinder or sphere, we introduce a slow time scale $\epsilon^{-2} \omega^{-1}$ inspired by the result of the previous numerical study. ${ }^{13}$ Thus, the analysis is accomplished with the method of multiple scales ${ }^{17,18}$ up to the third order, and a cubic nonlinear equation for complex wave amplitude is derived. Making use of the amplitude equation and its first integral, we explore several features of the modulation phenomenon.

We shall emphasize that the present study is not only a confirmation of the previous numerical simulation. It is known that the detuning can cause the amplitude modulation in the plane standing waves. ${ }^{19,20}$ However, the modulation resulting from detuning is essentially a linear wave phenomenon. This paper presents a mathematical evidence that the modulation in cylindrical and spherical standing waves is induced by the nonlinearity and geometrical effect irrespective of detuning.

\section{FORMULATION OF THE PROBLEM}

\section{A. Coaxial cylinders and concentric spheres}

We shall consider standing waves in an ideal gas between two infinitely long coaxial cylinders and two concentric spheres. The sound is generated in the gas by a harmonic oscillation of the radius of outer cylinder or sphere, given by

$$
r_{b}=\bar{r}_{b}-A_{0} \cos \omega t^{*},
$$

where $r_{b}$ is the instantaneous radius at time $t^{*}, \bar{r}_{b}$ is the mean radius, $A_{0}$ is an amplitude, and $\omega$ is an angular frequency of the harmonic oscillation (see Fig. 1). The inner cylinder or sphere is fixed and thus a standing acoustic wave with cylindrical or spherical symmetry is formed in the gas. If the driving frequency $\omega$ matches a resonance frequency of the system, the standing wave develops into a resonant oscillation. The problem can be described only by two independent variables, the time $t^{*}$ from the beginning of the oscillation, and the radial coordinate $r^{*}$ from the axis of the cylinders or the center of the spheres. Consequently, the two problems for cylindrical waves and spherical waves can be discussed in parallel.

In order to formulate the problem mathematically, the nondimensional variables are introduced:

$$
r=\frac{\omega r^{*}}{c_{0}}, \quad t=\omega t^{*}, \quad u=\frac{u^{*}}{c_{0}}, \quad \Phi=\frac{\Phi^{*} \omega}{c_{0}^{2}},
$$

where $c_{0}$ is the speed of sound in the initial undisturbed gas, $u^{*}$ is the radial component of the fluid velocity, $\Phi^{*}$ is the velocity potential defined by $u^{*}=\partial \Phi^{*} / \partial r^{*}$.

Now, the most important parameter related to the geometry of the problem is the radius ratio. The radius ratio $\alpha$ is defined as

$$
\alpha \equiv \frac{r_{a}}{\bar{r}_{b}} \quad(0 \leqq \alpha<1)
$$

where $r_{a}$ is the radius of inner cylinder or sphere. The parameter $\alpha$ can be regarded as a measure of geometrical effect. $^{21}$ In fact, when $\alpha$ is sufficiently small compared with unity, the wave amplitude at the inner boundary increases due to the focusing of cylindrical or spherical waves. The case that the inner cylinder or sphere does not exist is included in the present formulation as a limiting case of $\alpha \rightarrow 0$. On the other hand, if $\alpha$ becomes close to unity, with keeping the spacing between inner and outer boundaries constant, the wave motion becomes close to that of the plane wave. However, the treatment of the case of $\alpha \rightarrow 1$ requires some care, because the nonlinear plane wave resonance always leads to the shock formation if the dissipation effect is negligible, and the present method of analysis assumes the shock free resonant oscillations.

The acoustic Mach number $M$ at the outer cylinder or sphere is defined as

$$
M \equiv \frac{A_{0} \omega}{c_{0}} \quad(0<M \ll 1) .
$$

This parameter measures the magnitude of nonlinearity for the wave that has just been radiated from the sound source. As shown in the previous numerical study, ${ }^{13}$ the shock wave can be generated even if $\alpha$ is rather small, if $M$ is larger than the critical Mach number. In the present paper, we assume that $M$ is sufficiently small so as to not lead to the shock formation. The geometrical effect on the shock formation in nearly plane wave resonances has also been discussed by Ockendon et al. $^{22}$

By using the nondimensional variables defined above, the radius of outer and inner cylinder or sphere can be nondimensionalized as

$$
\text { mean radius of outer cylinder (or sphere): } r_{s}=\frac{\omega \bar{r}_{b}}{c_{0}},
$$

$$
\text { inner cylinder (of sphere): } \alpha r_{s}=\frac{\omega r_{a}}{c_{0}} \text {. }
$$

The parameter $r_{s}$ may be regarded as a nondimensional driving frequency. Note that $M$ should be sufficiently small so that an additional restriction $A_{0} \ll \bar{r}_{b}-r_{a}$, i.e., $M \ll(1-\alpha) r_{s}$, may be satisfied. 


\section{B. Governing equation}

The governing equation for the nondimensional velocity potential $\Phi(r, t)$ derived from mass, momentum, and energy conservation equations ${ }^{23}$ can be written as

$$
\begin{aligned}
\Delta \Phi-\frac{\partial^{2} \Phi}{\partial t^{2}}= & \frac{\partial}{\partial t}\left(\frac{\partial \Phi}{\partial r}\right)^{2}+\frac{1}{2} \frac{\partial \Phi}{\partial r} \frac{\partial}{\partial r}\left(\frac{\partial \Phi}{\partial r}\right)^{2} \\
& +(\gamma-1) \Delta \Phi\left[\frac{\partial \Phi}{\partial t}+\frac{1}{2}\left(\frac{\partial \Phi}{\partial r}\right)^{2}\right] \\
& -\frac{1}{\operatorname{Re}}\left(\frac{3}{4}+\frac{\mu_{v}}{\mu}+\frac{\gamma-1}{\operatorname{Pr}}\right) \frac{\partial}{\partial t} \Delta \Phi,
\end{aligned}
$$

where $\gamma, \mu$, and $\mu_{v}$ are (all assumed to be constant) the specific heat ratio of the ideal gas, coefficient of viscosity, and coefficient of bulk viscosity, respectively, and $\Delta$ is a Laplacian,

$$
\Delta= \begin{cases}\frac{1}{r} \frac{\partial}{\partial r}\left(r \frac{\partial}{\partial r}\right) & \text { for cylindrical waves } \\ \frac{1}{r^{2}} \frac{\partial}{\partial r}\left(r^{2} \frac{\partial}{\partial r}\right) & \text { for spherical waves. }\end{cases}
$$

The last term in the right-hand side of Eq. (7) represents the dissipation effect, and Re and Pr are Reynolds number ${ }^{24}$ and Prandtl number, respectively,

$$
\operatorname{Re} \equiv \frac{c_{0}^{2}}{\nu_{0} \omega}, \quad \operatorname{Pr} \equiv \frac{\nu_{0}}{\kappa_{0}},
$$

where $\nu_{0}$ and $\kappa_{0}$ are kinematic viscosity and thermal conductivity of initial undisturbed gas.

The boundary conditions are, in the nondimensional form, given by

$$
\begin{aligned}
& \frac{\partial \Phi}{\partial r}=0 \text { at } r=\alpha r_{s} \text { (inner boundary), } \\
& \frac{\partial \Phi}{\partial r}=M \sin t \text { at } r=r_{s}-M \cos t \text { (outer boundary), }
\end{aligned}
$$

where $r_{s}-M \cos t$ is the nondimensional instantaneous radius of the outer cylinder or sphere.

\section{MULTIPLE SCALES ANALYSIS}

Let us seek an approximate solution of Eq. (7) in the form of an asymptotic expansion with a small parameter $\epsilon$,

$$
\Phi=\epsilon \Phi_{1}(r, t, T)+\epsilon^{2} \Phi_{2}(r, t, T)+\epsilon^{3} \Phi_{3}(r, t, T)+O\left(\epsilon^{4}\right),
$$

where the expansion parameter $\epsilon$ is set as the order of the maximum wave amplitude, $M^{1 / 3}$,

$$
\epsilon \equiv M^{1 / 3},
$$

and a slow time variable $T$ defined by

$$
T \equiv \epsilon^{2} t=M^{2 / 3} t,
$$

is introduced as an independent variable in Eq. (12). The scaling, Eqs. (13) and (14), is motivated by the result of the previous numerical study. ${ }^{13}$ Thus we employ the method of multiple scales ${ }^{17}$ with $t$ and $T\left(=\epsilon^{2} t\right)$.

In addition, we also introduce a detuning parameter $\delta$ and a damping parameter $\eta$,

$$
r_{s}=r_{0}+\epsilon^{2} \delta, \quad \frac{1}{\operatorname{Re}}\left(\frac{3}{4}+\frac{\mu_{v}}{\mu}+\frac{\gamma-1}{\operatorname{Pr}}\right)=\epsilon^{2} \eta .
$$

Here, $r_{0}$ is a resonance radius correspond to the resonance frequency obtained from the linear wave equation (see the next subsection), $\epsilon^{2} \delta$ represents a distance between the resonance radius and the mean radius of the outer boundary called detuning, and $\epsilon^{2} \eta$ represents the dissipation effect. Equation (15) means that the detuning and damping are assumed to be comparable and very small. Actually, the damping coefficient $\eta$ is always small unless the driving frequency $\omega$ is quite large [see Eq. (9)] in nonlinear problems with small but finite $M$. For example, if $M \approx 10^{-3}, \omega \approx 10^{4}$, and the gas is the air $\left(\nu_{0} \approx 10^{-5} \mathrm{~m}^{2} / \mathrm{s}\right)$, then $\operatorname{Re} \approx 10^{6}$ and hence $\eta \approx \epsilon^{-2} \mathrm{Re}^{-1} \approx 10^{-4}$.

Substituting Eq. (12) into the governing equation (7) and boundary conditions (10) and (11), and equating coefficients of equal powers of $\epsilon$ leads to a sequence of linear problems for $\Phi_{n}(n=1,2,3, \ldots)$, which are successively solved in the subsequent subsections. Since the analysis in this section aims at deriving the equation for complex wave amplitude, which is capable of describing the modulation phenomenon, only the first- and second-order problems are solved exactly. In the third-order problem, the amplitude equation is derived from the so-called solvability condition to make the asymptotic expansion (12) uniformly valid.

\section{A. First-order problem}

The first-order problem is given by the linear wave equation

$$
\Delta \Phi_{1}-\frac{\partial^{2} \Phi_{1}}{\partial t^{2}}=0,
$$

and the boundary conditions

$$
\begin{aligned}
& \frac{\partial \Phi_{1}}{\partial r}=0 \text { at } r=\alpha r_{0}, \\
& \frac{\partial \Phi_{1}}{\partial r}=0 \text { at } r=r_{0},
\end{aligned}
$$

where the resonance radius $r_{0}\left(r_{0}>0\right)$ is to be determined.

By the method of separation of variables, the solution of the boundary value problem (16)-(18) is written in the form of a standing wave,

$$
\Phi_{1}(r, \theta, t)=A(T) \exp (i t) \phi_{1}(r)+\text { c.c. },
$$

where $i$ is the imaginary unit, $A$ is an unknown complex wave amplitude, $\phi_{1}$ is a real function of $r$, and c.c. implies the complex conjugate to all preceding terms. The functional form of $A$ with respect to the slow time variable $T$ is determined by the amplitude equation derived later.

The spatial profile $\phi_{1}$ satisfying the boundary condition (17) is 


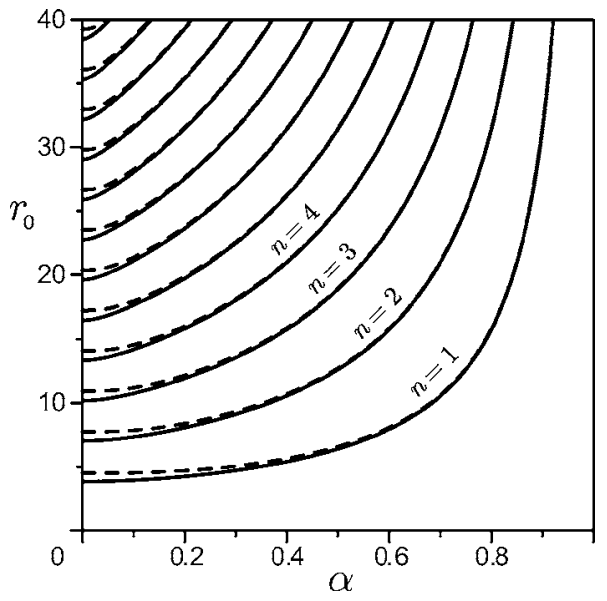

FIG. 2. The resonance radius $r_{0}$ calculated from (24) and (25). Solid curves denote those for cylindrical waves and dashed curves for spherical waves. The number near each curve signifies the mode number beginning from $n$ $=1$ for the fundamental; the number is omitted for $n>4$.

for cylindrical waves:

$$
\phi_{1}=\frac{J_{0}(r) N_{1}\left(\alpha r_{0}\right)-J_{1}\left(\alpha r_{0}\right) N_{0}(r)}{Z\left(\alpha r_{0}\right)},
$$

for spherical waves:

$$
\phi_{1}=\left(\alpha r_{0}\right)^{2}\left[S(r) C^{\prime}\left(\alpha r_{0}\right)-S^{\prime}\left(\alpha r_{0}\right) C(r)\right],
$$

where $J_{n}$ and $N_{n}(n=0,1)$ are Bessel and Neumann functions of order $n$. The denominator $Z\left(\alpha r_{0}\right)$ in Eq. (20) is defined as

$$
Z\left(\alpha r_{0}\right)=\sqrt{\left[J_{1}\left(\alpha r_{0}\right)\right]^{2}+\left[N_{1}\left(\alpha r_{0}\right)\right]^{2}},
$$

and introduced to eliminate the singularity of the numerator in the limit $\alpha \rightarrow 0$. The functions $S$ and $C$ in Eq. (21) are defined as

$$
S(r)=\frac{\sin r}{r}, \quad C(r)=\frac{\cos r}{r},
$$

and the prime denotes the derivative with respect to its argument. The singularity of the derivatives of $S$ and $C$ functions in the limit $\alpha \rightarrow 0$ is eliminated by a factor $\alpha^{2}$ in Eq. (21).

Applying boundary condition (18) to Eqs. (20) and (21) gives the resonance conditions for cylindrical waves,

$$
J_{1}\left(r_{0}\right) N_{1}\left(\alpha r_{0}\right)-J_{1}\left(\alpha r_{0}\right) N_{1}\left(r_{0}\right)=0,
$$

and for spherical waves,

$$
S^{\prime}\left(r_{0}\right) C^{\prime}\left(\alpha r_{0}\right)-S^{\prime}\left(\alpha r_{0}\right) C^{\prime}\left(r_{0}\right)=0,
$$

respectively. For a given $\alpha$, the resonance radius $r_{0}$ is determined as a solution of Eq. (24) or Eq. (25). In Fig. 2, the resonance radius of each $n$th mode $(n=1$ : the fundamental, $n=2,3, \ldots$ : higher modes) is plotted, where the solid curves denote those for cylindrical waves and the dashed curves for spherical waves. Except for small $\alpha(\alpha \lesssim 0.2)$, the difference of $r_{0}$ is small between the cylindrical and spherical waves.

\section{B. Second-order problem}

The second-order problem is given by

$$
\begin{aligned}
& \Delta \Phi_{2}-\frac{\partial^{2} \Phi_{2}}{\partial t^{2}}=\frac{\partial}{\partial t}\left(\frac{\partial \Phi_{1}}{\partial r}\right)^{2}+(\gamma-1) \frac{\partial^{2} \Phi_{1}}{\partial t^{2}} \frac{\partial \Phi_{1}}{\partial t}, \\
& \frac{\partial \Phi_{2}}{\partial r}=0 \text { at } r=\alpha r_{0} \text { and } r=r_{0} .
\end{aligned}
$$

Since the boundary condition (27) does not allow a steady component, we can put $\Phi_{2}$ in the form of the second harmonics

$$
\Phi_{2}=i A^{2} \exp (2 i t) \phi_{2}(r)+\text { c.c. },
$$

where $\phi_{2}$ is a real function of $r$.

Substituting Eq. (28) into Eqs. (26) and (27), we have a linear two-point boundary value problem for $\phi_{2}$, consisting of an inhomogeneous second-order ordinary differential equation and homogeneous Neumann boundary conditions at $r=\alpha r_{0}$ and $r=r_{0}$. As can be easily seen, the solvability condition for the boundary value problem for $\phi_{2}$ holds for an arbitrary $A$, and hence we can obtain the explicit forms of $\phi_{2}$ for the cylindrical wave,

$$
\begin{aligned}
\phi_{2}= & \pi \frac{J_{0}(2 r) N_{1}\left(2 \alpha r_{0}\right)-J_{1}\left(2 \alpha r_{0}\right) N_{0}(2 r)}{J_{1}\left(2 r_{0}\right) N_{1}\left(2 \alpha r_{0}\right)-J_{1}\left(2 \alpha r_{0}\right) N_{1}\left(2 r_{0}\right)} \\
& \times \int_{\alpha r_{0}}^{r_{0}}\left[J_{1}\left(2 r_{0}\right) N_{0}(2 \xi)-J_{0}(2 \xi) N_{1}\left(2 r_{0}\right)\right] \xi R_{2 C}(\xi) d \xi \\
& -\pi \int_{\alpha r_{0}}^{r}\left[J_{0}\left(2 r_{0}\right) N_{0}(2 \xi)-J_{0}(2 \xi) N_{0}\left(2 r_{0}\right)\right] \xi R_{2 C}(\xi) d \xi,
\end{aligned}
$$

where

$$
\begin{aligned}
R_{2 C}(\xi)= & {\left[J_{1}(\xi) N_{1}\left(\alpha r_{0}\right)-J_{1}\left(\alpha r_{0}\right) N_{1}(\xi)\right]^{2} } \\
& -\frac{\gamma-1}{2}\left[J_{0}(\xi) N_{1}\left(\alpha r_{0}\right)-J_{1}\left(\alpha r_{0}\right) N_{0}(\xi)\right]^{2},
\end{aligned}
$$

and for the spherical wave,

$$
\begin{aligned}
\phi_{2}= & -\frac{S(2 r) C^{\prime}\left(2 \alpha r_{0}\right)-S^{\prime}\left(2 \alpha r_{0}\right) C(2 r)}{S^{\prime}\left(2 r_{0}\right) C^{\prime}\left(2 \alpha r_{0}\right)-S^{\prime}\left(2 \alpha r_{0}\right) C^{\prime}\left(2 r_{0}\right)} \\
& \times \int_{\alpha r_{0}}^{r_{0}} \frac{2 r_{0} \cos 2\left(r_{0}-\xi\right)-\sin 2\left(r_{0}-\xi\right)}{4 r_{0}^{2}} \xi R_{2 S}(\xi) d \xi \\
& +\int_{\alpha r_{0}}^{r} \frac{\sin 2(r-\xi)}{2 r} \xi R_{2 S}(\xi) d \xi
\end{aligned}
$$

where

$$
\begin{aligned}
R_{2 S}(\xi)= & 2\left[S^{\prime}(\xi) C^{\prime}\left(\alpha r_{0}\right)-S^{\prime}\left(\alpha r_{0}\right) C^{\prime}(\xi)\right]^{2}-(\gamma-1) \\
& \times\left[S(\xi) C^{\prime}\left(\alpha r_{0}\right)-S^{\prime}\left(\alpha r_{0}\right) C(\xi)\right]^{2} .
\end{aligned}
$$

The calculation for deriving the explicit representation of $\phi_{2}$, Eqs. (29)-(32), is slightly tedious but the result is indispensable for the evaluation of coefficients of the amplitude equation in Sec. IV. 


\section{Third-order problem}

Finally, we proceed to the third-order problem, where the detuning and damping terms and the derivative with respect to the slow time variable $T$ first appear in the set of equations and boundary conditions,

$$
\begin{aligned}
\Delta \Phi_{3}-\frac{\partial^{2} \Phi_{3}}{\partial t^{2}}= & 2 \frac{\partial^{2} \Phi_{1}}{\partial t \partial T}-\eta \frac{\partial^{3} \Phi_{1}}{\partial t^{3}}+2 \frac{\partial}{\partial t}\left(\frac{\partial \Phi_{1}}{\partial r} \frac{\partial \Phi_{2}}{\partial r}\right) \\
& +\left(\frac{\partial \Phi_{1}}{\partial r}\right)^{2} \frac{\partial^{2} \Phi_{1}}{\partial r^{2}}+(\gamma-1)\left\{\frac { \partial ^ { 2 } \Phi _ { 1 } } { \partial t ^ { 2 } } \left[\frac{\partial \Phi_{2}}{\partial t}\right.\right. \\
& \left.\left.+\frac{1}{2}\left(\frac{\partial \Phi_{1}}{\partial r}\right)^{2}\right]+\Delta \Phi_{2} \frac{\partial \Phi_{1}}{\partial t}\right\} \\
\frac{\partial \Phi_{3}}{\partial r}=0 \text { at } r= & \alpha r_{0}, \quad \frac{\partial \Phi_{3}}{\partial r}=\sin t-\delta \frac{\partial^{2} \Phi_{1}}{\partial r^{2}} \text { at } r=r_{0} .
\end{aligned}
$$

Taking account of the fact that $\Phi_{1}$ is the fundamental and $\Phi_{2}$ is the second harmonics with respect to $t$, it is clear that the right-hand side of Eq. (33) is composed of only the fundamental and third harmonics. We can therefore put $\Phi_{3}$ in the form

$$
\Phi_{3}=\phi_{3}(r) \exp (i t)+F(3 r) \exp (3 i t)+\text { c.c. },
$$

where $\phi_{3}$ and $F$ are complex-valued.

Substituting Eq. (35) into Eqs. (33) and (34), after some manipulation, we have a linear boundary value problem for $\phi_{3}$,

$$
\begin{aligned}
& \frac{d}{d r}\left(r^{n} \frac{d \phi_{3}}{d r}\right)+r^{n} \phi_{3}=r^{n} R_{3}(r)+\text { c.c., } \\
& \frac{d \phi_{3}}{d r}=0 \text { at } r=\alpha r_{0}, \quad \frac{d \phi_{3}}{d r}=A \delta \phi_{1}-\frac{i}{2}+\text { c.c. at } r=r_{0},
\end{aligned}
$$

where $n=1$ and $n=2$, respectively, correspond to cylindrical and spherical waves, and

$$
\begin{aligned}
R_{3}(r)= & 2 i \frac{d A}{d T} \phi_{1}+A^{2} \bar{A}\left\{-2 \frac{d \phi_{1}}{d r} \frac{d \phi_{2}}{d r}+3\left(\frac{d \phi_{1}}{d r}\right)^{2} \frac{d^{2} \phi_{1}}{d r^{2}}\right. \\
& +(\gamma-1)\left[-2 \phi_{1} \phi_{2}+\frac{1}{2} \phi_{1}\left(\frac{d \phi_{1}}{d r}\right)^{2}\right] \\
& \left.-(\gamma-1)^{2} \phi_{1}^{3}\right\}
\end{aligned}
$$

$(\bar{A}$ is the complex conjugate of $A)$. From the solvability condition for the boundary value problem (36)-(38), we obtain a cubic nonlinear equation for complex wave amplitude $A$,

$$
i \hat{P}\left(2 \frac{d A}{d T}+\eta A\right)+\hat{Q} A^{2} \bar{A}+\hat{R} \delta A=\frac{i}{2} \hat{S}
$$

where coefficients $\hat{P}, \hat{Q}, \hat{R}$, and $\hat{S}$ are defined as follows:

$$
\begin{aligned}
\hat{P}= & \int_{\alpha r_{0}}^{r_{0}} r^{n} \phi_{1}^{2} d r \\
\hat{Q}= & \int_{\alpha r_{0}}^{r_{0}} r^{n} \phi_{1}\left\{-2 \frac{d \phi_{1}}{d r} \frac{d \phi_{2}}{d r}+3\left(\frac{d \phi_{1}}{d r}\right)^{2} \frac{d^{2} \phi_{1}}{d r^{2}}+(\gamma-1)\right. \\
& \left.\times\left[-2 \phi_{1} \phi_{2}+\frac{1}{2} \phi_{1}\left(\frac{d \phi_{1}}{d r}\right)^{2}\right]-(\gamma-1)^{2} \phi_{1}^{3}\right\} d r \\
\hat{R}= & -r_{0}^{n} \phi_{1}^{2}\left(r_{0}\right), \quad \hat{S}=-r_{0}^{n} \phi_{1}\left(r_{0}\right)
\end{aligned}
$$

The amplitude equation (39) is substantially equivalent to that of a forced oscillator with cubic nonlinearity. ${ }^{18}$ In Ref. 16 , another equivalent amplitude equation has been derived for an acoustic resonance in a tube with an array of Helmholtz resonators, with the analysis being focused on the steady state, i.e., $d A / d T=0$. A qualitative discussion of amplitude equations has also been given in a problem of resonance in nonuniform media. ${ }^{25}$

Once the oscillation mode is specified (e.g., the second mode of cylindrical waves, the third mode of spherical waves, and so on), and the radius ratio $\alpha$ is given, the resonance radius $r_{0}$ is obtained as a function of $\alpha$ by Eq. (24) or Eq. (25), as shown in Fig. 2. Then, all the coefficients $\hat{P}, \hat{Q}$, $\hat{R}$, and $\hat{S}$ can be determined from Eqs. (40)-(42), and the nonlinear response of complex amplitude $A$ can be examined by solving the amplitude equation (39). In the following, we investigate the behavior of the amplitude and phase responses in the resonances of cylindrical and spherical standing waves. The discussion will be focused on the fundamental mode, where the geometrical effect manifests itself most conspicuously.

\section{NONLINEAR RESPONSE OF THE RESONANT OSCILLATION AND THE MODULATION}

\author{
We shall introduce a polar form \\ $A(T)=a(T) \exp [i \theta(T)]$,
}

where $a(T)$ and $\theta(T)$ are real functions of $T$. In the polar form (43), $|a|$ is sometimes used instead of $a$. This does not make a substantial difference. We prefer the use of $a$ that makes $\theta$ continuous for $-\infty<\theta<\infty$. Substituting Eq. (43) into Eq. (39) and separating the real and imaginary parts, we have 

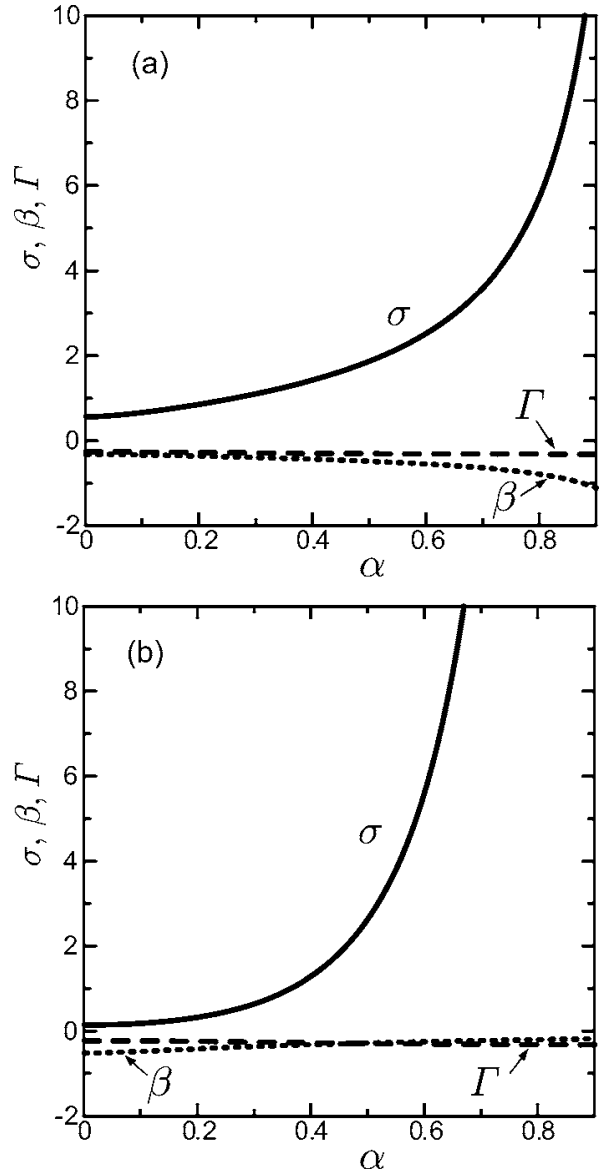

FIG. 3. The dependence of coefficients $\sigma, \beta$, and $\Gamma$ on $\alpha$. (a) Cylindrical waves; (b) spherical waves. Solid, dotted, and dashed lines denote $\sigma, \beta$, and $\Gamma$, respectively.

$$
a \frac{d \theta}{d T}=\sigma a^{3}-\beta \sin \theta+\Gamma \delta a
$$

$$
\frac{d a}{d T}=\beta \cos \theta-\frac{1}{2} \eta a,
$$

where

$$
\sigma=\frac{\hat{Q}}{2 \hat{P}}, \quad \beta=\frac{\hat{S}}{4 \hat{P}}, \quad \Gamma=\frac{\hat{R}}{2 \hat{P}} .
$$

The coefficients $\sigma, \beta$, and $\Gamma$ are constants depending only on $\alpha$, since $r_{0}$ is a function of $\alpha$ for a specified oscillation mode (see Fig. 2). In Fig. 3, $\sigma, \beta$, and $\Gamma$ are plotted as a function of $\alpha$ for the fundamental modes of cylindrical waves [Fig. 3(a)] and spherical waves [Fig. 3(b)]. As can be seen, $\beta$ and $\Gamma$ are negative, while $\sigma$ is positive for $0 \leqq \alpha \leqq 0.9$. Furthermore, $\sigma$ rapidly grows with the increase of $\alpha$. These parameters are essential for the analysis in the following.

\section{A. Resonant oscillation without damping}

If the dissipation effect is much smaller than the nonlinear effect, namely $\epsilon^{2} \operatorname{Re} \gg 1$ [see the second of Eq. (15)], the
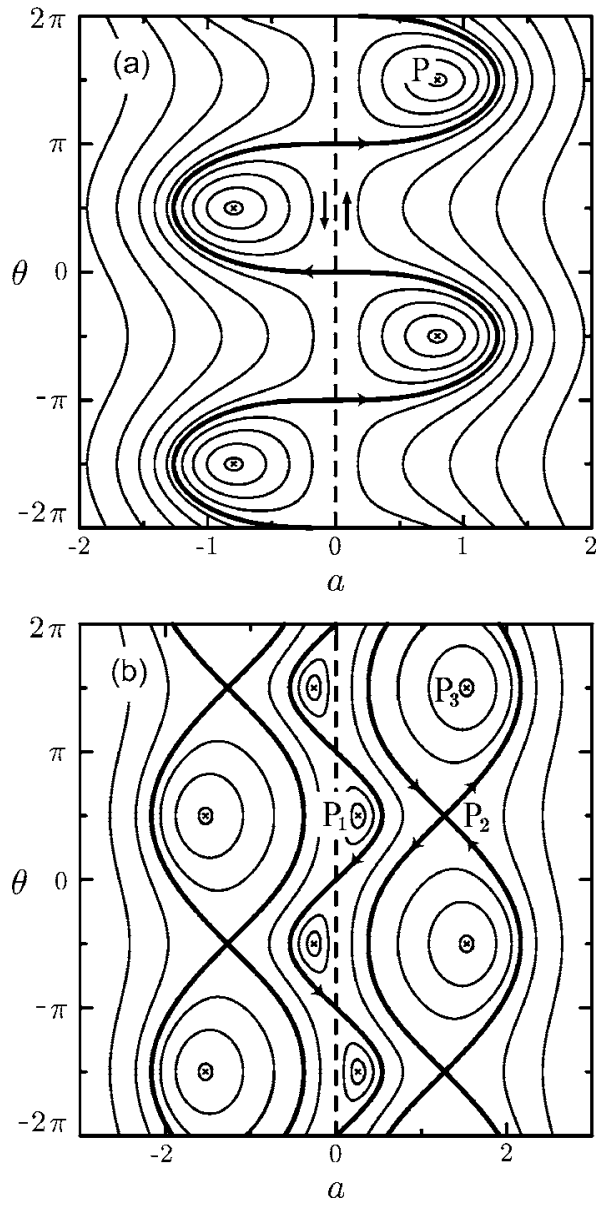

FIG. 4. Phase plane for the cylindrical wave problem with $\alpha=0.1$ and $\eta=0$. (a) $\delta=0$; (b) $\delta=5$. The symbol $\times$ denotes a fixed point (steady state oscillation); $P, P_{1}$, and $P_{3}$ are centers and $P_{2}$ is a saddle.

damping term with the factor $\eta$ may be neglected in Eq. (45). In this case, we can obtain a first integral of systems (44) and (45) with $\eta=0$.

When $\eta=0$, multiplying Eq. (44) by $\cos \theta$, multiplying Eq. (45) by $\sin \theta$, and adding up the resulting two equations, we have

$$
\frac{d(a \sin \theta)}{d T}=\left(\sigma a^{3}+\Gamma \delta a\right) \cos \theta .
$$

Dividing Eq. (47) by Eq. (45) with $\eta=0$ yields

$$
\frac{d(a \sin \theta)}{d a}=\frac{\sigma a^{3}+\Gamma \delta a}{\beta},
$$

and integrating Eq. (48), we obtain a first integral of systems (44) and (45) with $\eta=0$,

$$
a \sin \theta-\left(\frac{\sigma}{4 \beta} a^{4}+\frac{\Gamma \delta}{2 \beta} a^{2}\right)=C,
$$

where $C$ is an arbitrary constant determined by an initial data at $T=0$.

In Fig. 4, the contours of first integral (49) for the fundamental mode of cylindrical waves with $\alpha=0.1$ are shown in the $(a, \theta)$ plane, where Fig. 4(a) shows the exact resonance of $\delta=0$ and Fig. 4(b) shows the case of detuning $\delta=5$, and 




FIG. 5. The envelope of acoustic pressure at the surface of the inner cylinder for $\alpha=0.1$ and $\delta=\eta=0$. The solid curves are the solution of amplitude equation and the dashed curves are the numerical solution of Euler equations.

each curve represents a contour line of Eq. (49) for a given $C$. Since the entire set of curves in the $(a, \theta)$ plane is topologically equivalent to the entire set of trajectories in the $(a, d a / d T)$ plane, the $(a, \theta)$ plane may also be called the phase plane. Thick curves in the figures may be called the separatrix, which separates a region of closed trajectories from that of open trajectories. The symbol $\times$, a fixed point of system (44) and (45) with $\eta=0$, signifies a center or a saddle, given by

$$
\sigma a^{3}+\Gamma \delta a=\beta, \quad \theta=\frac{\pi}{2}+m \pi \quad(m=0, \pm 1, \pm 2, \ldots) .
$$

The fixed point corresponds to a steady state oscillation, and it has been examined, ${ }^{10-12}$ while other trajectories have been left untouched.

As can be seen from Fig. 4, the amplitude $a$ and the phase $\theta$ oscillate periodically along each closed trajectory, and also along each open trajectory, since $(a, \theta+2 \pi)$ can be identified with $(a, \theta)$ in the phase plane. This means that the resonant oscillation is subjected to a periodic modulation in the slow time scale $\epsilon^{-2} \omega^{-1}$. The steady state oscillation comes out only when the amplitude $a$ and the phase $\theta$ in an initial stage of the nonlinear process agree with a fixed point given by Eq. (50). Otherwise, the modulation necessarily appears irrespective of whether $\delta=0$ or not and of whether the trajectory in the phase plane is closed or open.

Here, it should be noted that the trajectory in the $(a, \theta)$ plane can cross the line $a=0$ only at $\theta=m \pi$ $(m=0, \pm 1, \pm 2, \ldots)$, and the flow directions of trajectories on the both sides of the line $a=0$ are opposite as indicated by arrows in Fig. 4(a); this is a result of the use of the polar form.

One of important trajectories is that passing through the line $a=0$ of the phase plane, because the resonant oscillation is usually started from an initial state uniform and at rest. The trajectory passing through the origin $(a, \theta)=(0,0)$ is a separatrix

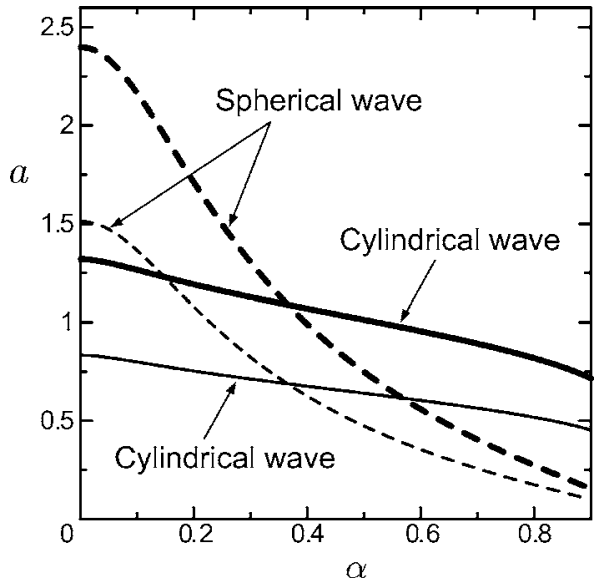

FIG. 6. The wave amplitude as a function of $\alpha$ for $\delta=\eta=0$. The thick solid and dashed curves are the maximum wave amplitudes of modulated resonant oscillations, while the thin solid and dashed curves are the wave amplitudes of steady state oscillations.

$$
\sin \theta=\frac{\sigma}{4 \beta} a^{3},
$$

for $\delta=0$, indicated by a thick curve in Fig. 4(a). In Fig. 5, we present the envelopes of acoustic pressure at the surface of the inner cylinder, where acoustic pressure $p^{\prime}$ is defined as the pressure fluctuation from the reference pressure, $p^{\prime}=p^{*}-p_{0}$. In the leading order of approximation in weakly nonlinear acoustics problems, the acoustic pressure is given by $p^{\prime}=-\rho_{0}\left(\partial \Phi^{*} / \partial t^{*}\right)$, and hence $p^{\prime} /\left(\rho_{0} c_{0}^{2}\right)$ $=2 \epsilon A(T) \phi_{1}(r) \sin t$. The solid curves in Fig. 5 denote $2 A(T) \phi_{1}\left(\alpha r_{0}\right)$ evaluated from the solution of Eq. (51) with Eq. (44), and dashed curves are the numerical solution of Euler equations for $M=10^{-4},{ }^{13}$ obtained by using a TVD finite-difference method. ${ }^{26}$ One can see that the wave motion corresponding to the separatrix agrees with the numerical solution started from an initial state of uniform and at rest. The difference between solid and dashed curves are caused by the higher-order nonlinear effects that are not included in systems (44) and (45).

Along the separatrix (51), an extremum of $a$ is reached at $\theta=\pi / 2+m \pi(m=0, \pm 1, \pm 2, \ldots)$. The maximum amplitude of the modulated oscillation when $\delta=\eta=0$ is therefore given by

$$
a_{\max }=\left(-\frac{4 \beta}{\sigma}\right)^{1 / 3},
$$

where $\sigma>0$ and $\beta<0$ (see Fig. 3). On the other hand, the amplitude of steady state oscillation for $\delta=\eta=0$ is immediately obtained from Eq. (50) as $a=(-\beta / \sigma)^{1 / 3}$. Figure 6 shows the dependence of $a_{\max }$ on $\alpha$, where thick solid and dashed lines indicate those in the fundamental modes of cylindrical and spherical waves, respectively. The amplitudes of steady state oscillations are also plotted with thin solid and dashed lines for cylindrical and spherical waves. All curves monotonically decrease with an increase in $\alpha$, and this is simply because $\sigma$ increases with an increase in $\alpha$ (see Fig. 3). The amplitude modulation is thus reduced as the wave motion approaches that in the plane wave, or in other words the 




FIG. 7. The dependence of period of modulation on the radius ratio $\alpha$. The solid curve denotes $T_{P}$ defined by Eq. (53), and the black circles denote those estimated from the numerical solution of Euler equations.

modulation is weakened as the geometrical effect decreases.

Substituting Eq. (51) into Eq. (44) with $\delta=0$, and integrating the result, we can evaluate the period of modulation $T_{P}$ along the separatrix passing through the origin as

$$
T_{P}=\frac{4}{3}\left(\frac{4}{\sigma \beta^{2}}\right)^{1 / 3} \int_{0}^{\pi / 2} \frac{d \theta}{\sin ^{2 / 3} \theta}=\frac{2[\Gamma(1 / 6)]^{2}}{3\left(\sigma \beta^{2}\right)^{1 / 3} \Gamma(1 / 3)},
$$

with the help of a formula ${ }^{27}$

$$
\int_{0}^{\pi / 2} \sin ^{\mu-1} x d x=2^{\mu-2} B\left(\frac{\mu}{2}, \frac{\mu}{2}\right),
$$

where $\Gamma(p)$ is the Gamma function and $B(q, r)$ is the beta function. Figure 7 shows the dependence of the period $T_{P}$ of amplitude modulation on the radius ratio $\alpha$ for the fundamental mode of cylindrical standing waves for $\delta=\eta=0$. Black circles denote the results of numerical solutions of Euler equations. ${ }^{13}$ Clearly, the modulation period decreases monotonically with increasing $\alpha$.

The trajectories in Fig. 4(b) are those for detuning $\delta=5$, where two centers $\left(P_{1}\right.$ and $\left.P_{3}\right)$ are separated by two types of separatrices passing through a saddle $\left(P_{2}\right)$ and the origin. These three fixed points explains the frequency response of the steady state oscillation. ${ }^{10-12}$ Even in the case of detuning, as long as the dissipation effect is negligible, the resonant oscillation starting from the initial quiescent gas experiences the periodic modulation along the separatrix passing through the origin.

\section{B. Resonant oscillation with damping}

As mentioned in the beginning of Sec. III, the damping coefficient $\eta$ is usually small. However, if the driving frequency $\omega$ is quite large, say $\omega \approx 10^{7}$, and $M \approx 10^{-3}$, then $\eta \approx 0.1$ in the air. We now deal with such a relatively large damping effect on the resonant oscillations.

Figure 8 shows the trajectories in the phase plane for the fundamental mode of cylindrical standing waves with $\alpha$ $=0.1, \eta=0.3$, and $\delta=0$ [Fig. $8(\mathrm{a})]$ or $\delta=5$ [Fig. 8(b)]. It can be seen from the figure that trajectories spiral into a fixed point, which is easily verified as a stable focus of systems
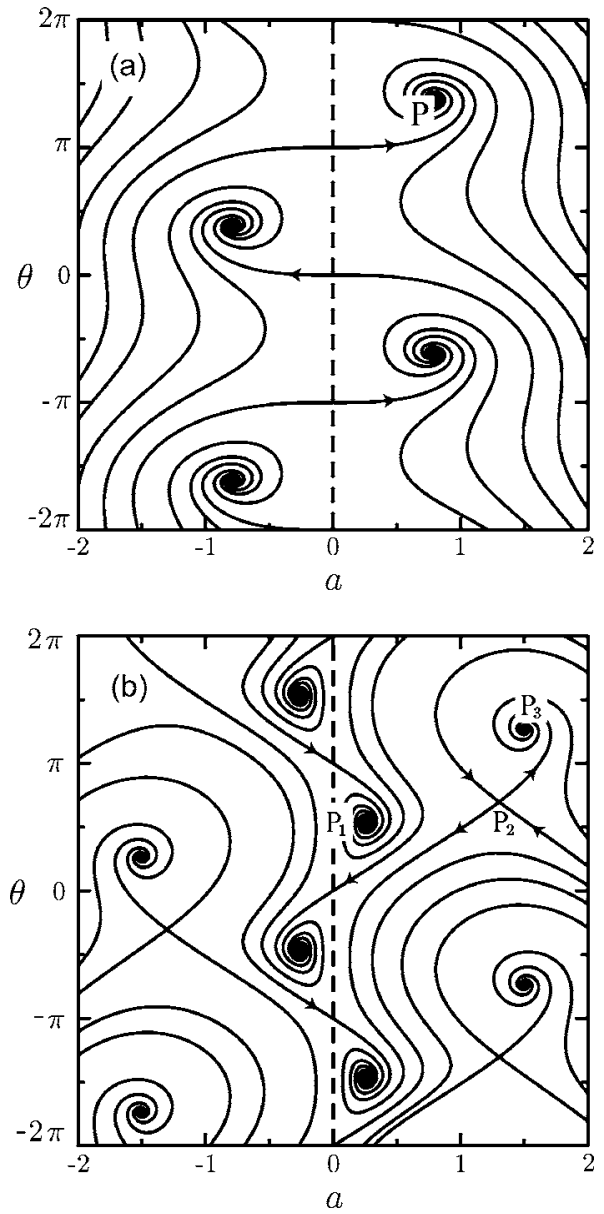

FIG. 8. Phase plane for the cylindrical wave problem with $\alpha=0.1$ and $\eta=0.3$. (a) $\delta=0$; (b) $\delta=5$. The symbols $P, P_{1}$, and $P_{3}$ are stable foci and $P_{2}$ is a saddle.

(44) and (45) with $\eta \neq 0$. That is, the modulation of amplitude and phase decay with time due to the dissipation effect, and finally, the resonant oscillation reaches a steady state oscillation. In the figure, the trajectory issuing from the origin is immediately attracted to the focus at $\theta \approx \pm \pi / 2$, since the damping effect is relatively large.

The amplitude response of the steady state oscillation can be examined by the amplitude equation (or fixed point equation)

$$
\left(\sigma a^{2}+\Gamma \delta\right)^{2}-\frac{\beta^{2}}{a^{2}}+\frac{\eta^{2}}{4}=0,
$$

which is derived by setting $d a / d T=d \theta / d T=0$ in Eqs. (44) and (45). Figure 9 shows the amplitude response of steady state oscillation for varying $\delta$ for the fundamental mode of the cylindrical waves with $\alpha=0.1$. The symbols $P, P_{1}, P_{2}$, and $P_{3}$, respectively, correspond to fixed points shown in Figs. 4 and 8. As can be seen, the amplitude response curve is hardly affected by the dissipation effect, while the trajectories in the phase plane for $\eta=0.3$ are drastically changed from those in the case of $\eta=0$ (see Figs. 4 and 8). Since Eq. (55) can be rewritten into a bicubic equation for $a$, the number of positive solutions $a$ can be examined by making use of the analysis presented in Appendices A and B. The result for 


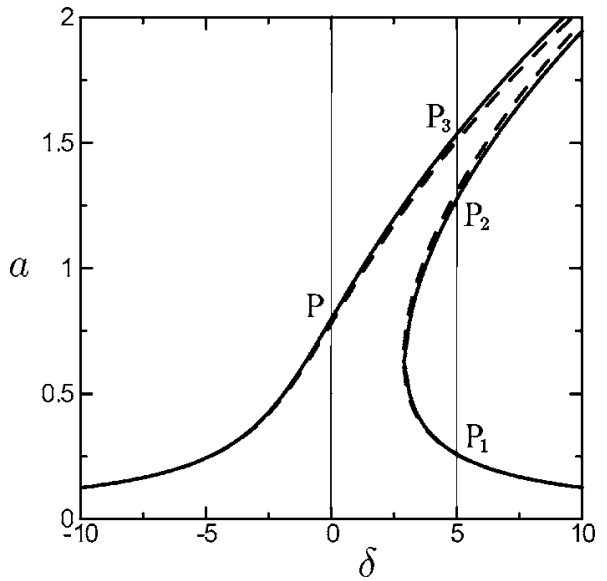

FIG. 9. Amplitude response in the steady state oscillations. Solid and dashed curves denote those for $\eta=0$ and $\eta=0.3$, respectively.

the cylindrical wave for $\eta=0$ is shown in Fig. 10. The region of three solutions (one is a saddle, the others are centers) decreases with the increase of $\alpha$.

\section{CONCLUSION}

We have theoretically studied the weakly nonlinear shock free resonant oscillation with cylindrical or spherical symmetry generated between two coaxial cylinders or two concentric spheres. By applying the method of multiple scales, the cubic nonlinear amplitude equation has been derived as well as the explicit representations of its coefficients. Then, we have examined a first integral of the amplitude equation for $\eta=0$, thereby demonstrating that the resonant oscillation is accompanied by the periodic modulation. The period of modulation has also been obtained explicitly for the oscillation beginning from the initial quiescent inviscid gas. Furthermore, the brief discussion on the dissipation effect has been given.

Since the modulation of shock free cylindrical and spherical standing waves is caused by the combination of nonlinear and geometrical (dissonant) effects, it is essential to address how the modulation is affected by the geometrical effect. The problem setup of two coaxial cylinders and two concentric spheres and introduction of the parameter $\alpha$ have enabled us to clarify the geometrical effect.

The studies on the acoustic resonances of spherical and cylindrical symmetric waves have so far been limited to the steady state oscillations in a sphere and in a cylinder without the inner boundary. By the present theoretical analysis and the previous numerical study, we have completed the problem with spherical or cylindrical symmetry. The analysis of a resonant oscillation between two eccentric cylinders, where an asymmetric mode of the standing waves is excited, is now underway. ${ }^{28}$

\section{APPENDIX A: STEADY STATE OSCILLATION}

The amplitude of the steady state oscillation can be found as a solution of Eq. (55) or the following bicubic equation:

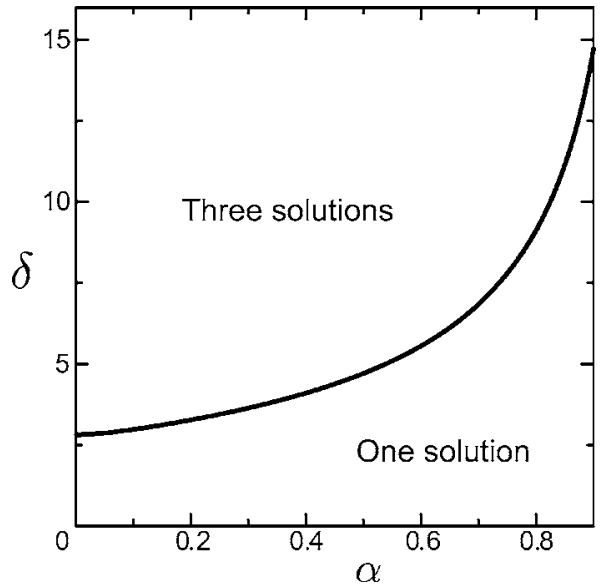

FIG. 10. The number of solutions for the steady state oscillation of the fundamental mode of cylindrical standing waves with $\eta=0$.

$$
\sigma^{2} a^{6}+2 \sigma \Gamma \delta a^{4}+\left(\Gamma^{2} \delta^{2}+\frac{\eta^{2}}{4}\right) a^{2}-\beta^{2}=0 .
$$

Introducing expressions,

$\mathcal{A}^{\prime}=\frac{2 \Gamma \delta}{\sigma}, \quad \mathcal{B}^{\prime}=\frac{1}{\sigma^{2}}\left(\Gamma^{2} \delta^{2}+\frac{\eta^{2}}{4}\right), \quad \mathcal{C}^{\prime}=-\frac{\beta^{2}}{\sigma^{2}}, \quad x=a^{2}$,

we can rewrite Eq. (A1) into a cubic equation as

$$
f(x)=x^{3}+\mathcal{A}^{\prime} x^{2}+\mathcal{B}^{\prime} x+\mathcal{C}^{\prime}=0 .
$$

By means of Cardano's formula, a solution for Eq. (A2) is given as

$$
x=X_{n}-\frac{1}{3} \mathcal{A}(n=1,2,3), \quad \mathcal{A}=\mathcal{B}^{\prime}-\frac{\mathcal{A}^{\prime}}{3},
$$

where $X_{n}$ is defined by

$$
\begin{aligned}
& X_{1}=u_{1}+v_{1}, \quad X_{2}=\omega u_{1}+\omega^{2} v_{1}, \quad X_{3}=\omega^{2} u_{1}+\omega v_{1}, \\
& u_{1}=\sqrt[3]{\frac{\mathcal{B}}{2}+\sqrt{D}}, \quad v_{1}=\sqrt[3]{\frac{\mathcal{B}}{2}-\sqrt{D}},
\end{aligned}
$$

with $\omega=(-1 \pm \sqrt{3} i) / 2$ and

$$
\mathcal{B}=\frac{2 \mathcal{A}^{\prime 3}}{27}-\frac{\mathcal{A}^{\prime} \mathcal{B}^{\prime}}{3}+\mathcal{C}^{\prime}, \quad D=\frac{\mathcal{B}^{2}}{4}+\frac{\mathcal{A}^{3}}{27} \text {. }
$$

If $D<0$, Eq. (A2) has three distinct real solutions. In contrast, when $D \geqslant 0$, Eq. (A2) has only one real solution.

In particular, when $\delta=\eta=0$ (in this case, $D$ is always positive), Eq. (A2) is reduced to $\sigma a^{3}=\beta$, and hence we have $a=(-\beta / \sigma)^{1 / 3}$.

\section{APPENDIX B: STEADY STATE OSCILLATION IN INVISCID GAS}

The explicit representation for the amplitude of the steady state oscillation for $\eta=0$ can be obtained by making use of Cardano's formula shown in Appendix A. Here, we note that in the case of $\eta=0$,

$$
\mathcal{B}=-\frac{1}{\sigma}\left(\frac{2 \Gamma^{3} \delta^{3}}{27 \sigma}+\beta^{2}\right),
$$




$$
D=\frac{\beta^{2}}{\sigma^{4}}\left(\frac{\Gamma^{3} \delta^{3}}{27 \sigma}+\frac{\beta^{2}}{4}\right)
$$

where $\sigma>0, \beta<0$, and $\Gamma<0$ (see Fig. 3).

\section{1. $D<0$ (Three distinct real solutions)}

In the case of $D<0, u_{1}$ and $v_{1}$ are complex numbers. From Eq. (B2), $\delta$ should be positive because $\sigma>0$ and $\Gamma<0 ; \mathcal{A}^{\prime}$ is therefore negative when $D<0$. Hence, we can write $\sqrt{D}=\sqrt{|D|} i$ and then

$$
\begin{aligned}
\left|u_{1}^{3}\right|=\left|v_{1}^{3}\right|= & \sqrt{\left(-\frac{\mathcal{B}}{2}\right)^{2}+|D|}=\sqrt{\left(-\frac{\mathcal{B}}{2}\right)^{2}-D,} \\
\left(-\frac{\mathcal{B}}{2}\right)^{2}-D= & \frac{1}{4 \sigma^{4}}\left(\frac{4 \Gamma^{6} \delta^{6}}{27^{2} \sigma^{2}}+\frac{4 \Gamma^{3} \delta^{3} \beta^{2}}{27 \sigma}+\beta^{4}\right) \\
& -\frac{\beta^{2}}{\sigma^{4}}\left(\frac{\Gamma^{3} \delta^{3}}{27 \sigma}+\frac{\beta^{4}}{4}\right)=\left(\frac{\Gamma \delta}{3 \sigma}\right)^{6}, \\
\therefore\left|u_{1}\right|=\left|v_{1}\right|=\mid & \left|\frac{\Gamma \delta}{3 \sigma}\right| .
\end{aligned}
$$

According to an inequality

$$
\left|u_{1}+v_{1}\right| \leqslant\left|u_{1}\right|+\left|v_{1}\right|=\left|\frac{2 \Gamma \delta}{3 \sigma}\right|=\left|\frac{\mathcal{A}^{\prime}}{3}\right|,
$$

it is found that $x$ is always positive,

$$
x=X_{1}-\frac{\mathcal{A}^{\prime}}{3}=u_{1}+v_{1}-\frac{\mathcal{A}^{\prime}}{3} \geqslant 0 .
$$

Similar discussions can be made for $X_{2}$ and $X_{3}$.

\section{2. $D \geqslant 0$ (Only one real solution)}

In this case, $\mathcal{B}$ defined by Eq. (B1) is always negative, and therefore $-\mathcal{B} / 2$ is positive. Moreover, from a relation,

$$
\left(-\frac{\mathcal{B}}{2}\right)^{2}-D=\left(\frac{\Gamma \delta}{3 \sigma}\right)^{6} \geqslant 0
$$

it can be seen that both $u_{1}$ and $v_{1}$ are positive. Therefore, by the arithmetic-geometric mean inequality,

$$
\begin{aligned}
& u_{1}+v_{1}=\sqrt[3]{-\frac{\mathcal{B}}{2}+\sqrt{D}}+\sqrt[3]{-\frac{\mathcal{B}}{2}-\sqrt{D}} \\
& \geqslant 2 \cdot \sqrt[6]{\left(-\frac{\mathcal{B}}{2}\right)^{2}-D}=2 \cdot \sqrt[6]{\left(\frac{\Gamma \delta}{3 \sigma}\right)^{6}}, \\
& \therefore u_{1}+v_{1} \geqslant \frac{1}{3}\left|\frac{2 \Gamma \delta}{\sigma}\right|=\left|\frac{\mathcal{A}^{\prime}}{3}\right|>0 .
\end{aligned}
$$

In the case of $\delta \geqslant 0, \mathcal{A}^{\prime}$ is negative because of $\sigma>0$ and $\Gamma<0$, and hence

$$
\begin{aligned}
& u_{1}+v_{1}>-\frac{\mathcal{A}^{\prime}}{3}>0, \\
& \therefore x=X_{1}-\frac{\mathcal{A}^{\prime}}{3}=u_{1}+v_{1}-\frac{\mathcal{A}^{\prime}}{3}>-\frac{2}{3} \mathcal{A}^{\prime}>0 .
\end{aligned}
$$

In the case of $\delta<0$, since $\mathcal{A}^{\prime}$ is positive, from Eq. (B5),

$$
\begin{aligned}
& u_{1}+v_{1}>\frac{\mathcal{A}^{\prime}}{3}, \\
& \therefore x=X-\frac{\mathcal{A}^{\prime}}{3}=u_{1}+v_{1}-\frac{\mathcal{A}^{\prime}}{3}>0 .
\end{aligned}
$$

From Eqs. (B3), (B6), and (B7), it can be seen that Eq. (A2) has only one real solution for $D \geqslant 0$ and three distinct real solutions for $D<0$, and moreover, these solutions are always positive independent of the sign of $D$.

${ }^{1}$ R. Betchov, "Nonlinear oscillations of a column of gas," Phys. Fluids 1, 205 (1958).

${ }^{2}$ W. Chester, "Resonant oscillations in closed tubes," J. Fluid Mech. 18, 44 (1964).

${ }^{3}$ S. Temkin, "Nonlinear gas oscillation in a resonant tube," Phys. Fluids 11, 960 (1968).

${ }^{4}$ B. R. Seymour and M. P. Mortell, "Resonant acoustic oscillations with damping: small rate theory," J. Fluid Mech. 58, 353 (1973).

${ }^{5} \mathrm{~N}$. Rott, "The heating effect connected with non-linear oscillations in a resonance tube," Z. Angew. Math. Phys. 25, 619 (1974).

${ }^{6}$ R. G. Zaripov and M. A. Ilhamov, "Non-linear gas oscillations in a pipe," J. Sound Vib. 46, 245 (1976).

${ }^{7}$ M. Ochmann, "Nonlinear resonant oscillations in closed tubes-An application of the averaging method," J. Acoust. Soc. Am. 77, 61 (1985).

${ }^{8}$ A. Goldshtein, P. Vainshtein, M. Fichman, and C. Gutfinger, "Resonant gas oscillations in closed tubes," J. Fluid Mech. 322, 147 (1996).

${ }^{9} \mathrm{~T}$. Yano, "Turbulent acoustic streaming excited by resonant gas oscillation with periodic shock waves in a closed tube," J. Acoust. Soc. Am. 106, L7 (1999).

${ }^{10} \mathrm{~W}$. Chester, "Acoustic resonance in spherically symmetric waves," Proc. R. Soc. London 434, 459 (1991).

${ }^{11}$ W. Ellermeier, "Acoustic resonance of cylindrically symmetric waves," Proc. R. Soc. London 445, 181 (1994).

${ }^{12} \mathrm{~W}$. Ellermeier, "Acoustic resonance of radially symmetric waves in a thermoviscous gas," Acta Mech. 121, 97 (1997).

${ }^{13}$ E. Kurihara, Y. Inoue, and T. Yano, "Nonlinear resonant oscillations and shock waves generated between two coaxial cylinders," Fluid Dyn. Res. 36, 45 (2004).

${ }^{14}$ A. B. Coppens and A. A. Atchley, "Nonlinear standing waves in cavities," in Encyclopedia of Acoustics, edited by M. J. Crocker (Wiley, New York, 1997), Vol. 1, pp. 237-247. 
${ }^{15}$ C. Lawrenson, B. Lipkens, T. S. Lucas, D. K. Perkins, and T. W. Van Doren, "Measurements of macrosonic standing waves in oscillating closed cavity," J. Acoust. Soc. Am. 104, 623 (1998).

${ }^{16}$ N. Sugimoto, M. Masuda, and T. Hashiguchi, "Frequency response of nonlinear oscillations of air column in a tube with an array of Helmholtz resonators," J. Acoust. Soc. Am. 114, 1772 (2003).

${ }^{17}$ A. Nayfeh, Perturbation Methods (Wiley, New York, 1973).

${ }^{18}$ A. Nayfeh and D. T. Mook, Nonlinear Oscillations (Wiley, New York, 1979).

${ }^{19}$ S. A. Egorushkin and A. N. Troshko, "Near-resonance highly nonlinear gas oscillations in tubes," Fluid Dyn. 29, 561 (1994).

${ }^{20}$ A. A. Aganin, M. A. Ilgamov, and E. T. Smirnova, "Development of longitudinal gas oscillations in a closed tube," J. Sound Vib. 195, 359 (1996).

${ }^{21}$ There is another option for the parameter for geometrical effect, i.e., the ratio of the spacing between the outer and inner boundaries to the mean radius of the outer boundary, $\left(\bar{r}_{b}-r_{a}\right) / \bar{r}_{b}$, and this is equal to $1-\alpha$.
${ }^{22}$ H. Ockendon, J. R. Ockendon, M. R. Peake, and W. Chester, "Geometrical effects in resonant gas oscillations," J. Fluid Mech. 257, 210 (1993).

${ }^{23}$ D. G. Crighton, "Model equations of nonlinear acoustics," Annu. Rev. Fluid Mech. 11, 11 (1979).

${ }^{24}$ In nonlinear acoustics, the acoustic Reynolds number defined by $c_{0} u_{0} /\left(\nu_{0} \omega\right)$ is often used instead of Re defined in Eq. (9), where $u_{0}$ is a typical fluid velocity.

${ }^{25}$ W. Ellermeier, "Nonlinear acoustics in non-uniform infinite and finite layers," J. Fluid Mech. 257, 183 (1993).

${ }^{26}$ S. R. Chakravarthy, "Development of upwind schemes for the Euler equations," NASA Contractor Report No. 4043 (1987).

${ }^{27}$ I. S. Gradshteyn and I. M. Ryzhik, Table of Integral, Series, and Products, 6th ed. (Academic, San Diego, 2000), p. 3.621.1.

${ }^{28}$ E. Kurihara and T. Yano, "Nonlinear resonant oscillations generated between two coaxial or eccentric cylinders," AIP Conf. Proc. 838, 461 (2006). 